\title{
Interferences between Bogoliubov excitations in superfluids of light
}

\author{
Quentin Fontaine, ${ }^{1}$ Pierre-Élie Larré, ${ }^{2}$ Giovanni Lerario, ${ }^{1,3}$ Tom Bienaimé, ${ }^{1}$ Simon Pigeon, ${ }^{1}$ Daniele Faccio, ${ }^{4}$ \\ Iacopo Carusotto $\odot,{ }^{5}$ Élisabeth Giacobino, ${ }^{1}$ Alberto Bramati, ${ }^{1}$ and Quentin Glorieux $\oplus^{1, *}$ \\ ${ }^{1}$ Laboratoire Kastler Brossel, Sorbonne Université, Centre National de la Recherche Scientifique, ENS-PSL Research University, \\ Collège de France, Paris 75005, France \\ ${ }^{2}$ CY Cergy Paris Université, Centre National de la Recherche Scientifique, LPTM, F-95000 Cergy, France \\ ${ }^{3}$ Consiglio Nazionale delle Ricerche NANOTEC Institute of Nanotechnology, Via Monteroni, 73100 Lecce, Italy \\ ${ }^{4}$ School of Physics and Astronomy, University of Glasgow, Glasgow G12 8QQ, Scotland, United Kingdom \\ ${ }^{5}$ INO-Consiglio Nazionale delle Ricerche BEC Center and Università di Trento, Via Sommarive, 38123 Povo, Italy
}

(Received 16 July 2020; accepted 26 October 2020; published 1 December 2020)

\begin{abstract}
Paraxial fluids of light represent an alternative platform to atomic Bose-Einstein condensates and superfluid liquids for the study of the quantum behavior of collective excitations. A key step in this direction is the precise characterization of the Bogoliubov dispersion relation, as recently shown in two experiments. However, the predicted interferences between the phonon excitations that would be a clear signature of the collective superfluid behavior have not been observed to date. Here, by analytically, numerically, and experimentally exploring the phonon phase velocity, we observe the presence of interferences between counterpropagating Bogoliubov excitations and demonstrate their critical impact on the measurement of the dispersion relation. These results are evidence of a key signature of light superfluidity and provide a characterization tool for quantum simulations with photons.
\end{abstract}

DOI: 10.1103/PhysRevResearch.2.043297

\section{INTRODUCTION}

The weakly beyond-mean-field description of a Bose quantum fluid, initially introduced by Bogoliubov, relies on small collective excitations on top of a time-independent condensate $[1,2]$. These excitations are described as noninteracting quasiparticles with a specific energy spectrum: soundlike at low momenta and free-particle-like at large momenta. Due to these linear then parabolic dependences at, respectively, low and large momenta, a system exhibiting this type of dispersion satisfies the Landau criterion for superfluidity [3], which is a benchmark for the system to behave as a superfluid, one of the most striking manifestations of quantum many-body physics.

In optics, a growing community focuses on quantum-fluid physics with light in nonlinear media [4]. For example, Bose-Einstein condensation has been observed both in exciton-polariton [5] and dye-filled [6] microcavities. Initially proposed by Pomeau and Rica [7] and neglected experimentally for a long time, paraxial fluids of light present exciting perspectives for studying quantum-fluids physics $[8,9]$. In this approach, photons acquire an effective mass as a consequence of the paraxial approximation while effective repulsive photon-photon interactions are mediated by

\footnotetext{
*Corresponding author: quentin.glorieux@lkb.upmc.fr
}

Published by the American Physical Society under the terms of the Creative Commons Attribution 4.0 International license. Further distribution of this work must maintain attribution to the author(s) and the published article's title, journal citation, and DOI. the optical nonlinearity of the medium in which they propagate. Experimental implementations rely on the propagation of an intense laser beam within a negative third-order, Kerr nonlinear medium such as photorefractive crystals [10,11], thermo-optic media [12,13], and hot atomic vapors $[14,16]$. In this $(2+1)$-dimensional geometry, the system is two dimensional (2D) in the transverse direction and the propagation coordinate is analogous to an effective time.

Recently, two experiments have measured the dispersion relation of weak-amplitude excitations on top of a paraxial fluid of light with two complementary approaches $[15,16]$ following a proposal of Ref. [8]. The evolution of these elementary excitations is described by the Bogoliubov theory, revealing the rich analogy existing between nonlinear photonics and quantum condensed matter physics. If this analogy is now well established, theoretical works $[17,18]$ have questioned the presence and the impact of interferences between counterpropagating Bogoliubov excitations in paraxial fluids of light. In this paper, we present experimental evidence of these interferences and we demonstrate their dramatic impact on the reconstruction of the dispersion relation and on the identification of superfluidity of light. Moreover, we propose an interpretation of these interferences as stimulated analogs of the Sakharov oscillations of cosmology [19,20], recently observed in an atomic condensate [21].

Finally, we show that this effect is robust across several experimental systems used for paraxial fluids of light by numerically taking into account the photon absorption, the finite size of the fluid, the saturation, and the nonlocality of the photon-photon interactions. Because all these corrections only marginally impact the observed behavior, 
our paper opens the way to novel experimental techniques for probing paraxial fluid of light, based on the observation of Bogoliubov-excitation interferences. For example, we propose that extracting the contrast of constructive interference fringes in the output plane as a function of the probe parameters will give access to the efficiency at which we can excite phonons, also known as the static structure factor [22].

\section{PARAXIAL FLUID OF LIGHT}

We consider a monochromatic beam of light propagating along the positive- $z$ direction in a $\chi^{(3)}$ nonlinear medium. In the paraxial and scalar approximations, the evolution of the slowly varying envelope $\mathcal{E}\left(\mathbf{r}_{\perp}, z\right)$ of the complex electric field $E\left(\mathbf{r}_{\perp}, z\right)=\mathcal{E}\left(\mathbf{r}_{\perp}, z\right) e^{i\left(k_{0} z-\omega t\right)}$ is known to obey the nonlinear Schrödinger equation (NLSE) of nonlinear optics [23]:

$$
i \frac{\partial \mathcal{E}}{\partial z}=\left(-\frac{1}{2 k_{0}} \nabla_{\perp}^{2}-\frac{3 k_{0} \chi^{(3)}}{8 n^{2}}|\mathcal{E}|^{2}-\frac{i \alpha}{2}\right) \mathcal{E} .
$$

In this equation, $k_{0}=n \omega / c$ is the laser propagation constant in the medium with $n$ the linear refractive index, $\omega$ the laser angular frequency, and $c$ the vacuum speed of light; $\nabla_{\perp}$ is the gradient with respect to the transverse coordinates $\mathbf{r}_{\perp}=$ $(x, y)$; and $\alpha \geqslant 0$ is the absorption coefficient describing photon losses.

Except for the last term describing losses, (1) is formally analogous to the Gross-Pitaevskii equation (GPE) describing the temporal evolution of the macroscopic wave function of an atomic Bose-Einstein condensate (BEC) in two dimensions [2]. In the right-hand side in particular, the Laplacian term mimics the kinetic-energy term with a mass corresponding to the laser propagation constant $k_{0}$. In Eq. (1), there is no external potential acting as a trapping term since the transverse beam expansion is assumed to be small. This hypothesis will be later validated in the discussion of Fig. 5(b). On the other hand, the $\chi^{(3)}$ contribution is analogous to the contact-interaction potential with an interaction parameter $g$ proportional to the Kerr susceptibility: $g=-3 k_{0} \chi^{(3)} /\left(8 n^{2}\right)$. In the following, we consider the nonlinearity to be self-defocusing $\left(\chi^{(3)}<0\right)$ so that the effective photon-photon interactions are repulsive $(g>0)$. In this analogy, the fluid density $\rho\left(\mathbf{r}_{\perp}, z\right)$ is directly proportional to the field intensity $I\left(\mathbf{r}_{\perp}, z\right)$ according to $\rho\left(\mathbf{r}_{\perp}, z\right)=\left|\mathcal{E}\left(\mathbf{r}_{\perp}, z\right)\right|^{2}=$ $2 I\left(\mathbf{r}_{\perp}, z\right) /\left(c \epsilon_{0} n\right)$, where $\epsilon_{0}$ denotes the vacuum permittivity. However, while the GPE describes the evolution of a condensate wave function for a matter quantum fluid in time, the NLSE describes how the electric-field envelope $\mathcal{E}\left(\mathbf{r}_{\perp}, z\right)$ of the light beam propagates in space, along the $z$ axis. Therefore, the propagation coordinate $z$ is equivalent to an effective time in the NLSE. As a consequence, every transverse plane (spanned by $\mathbf{r}_{\perp}$ ) along the propagation axis $z$ can be regarded as a snapshot of the evolution of the two-dimensional paraxial fluid of light (see Fig. 1). The role of the physical time $t$ as a third spatial coordinate for propagating light was highlighted in Ref. [24]. These features are, however, not relevant in the monochromatic excitation case under investigation here.

In the following theoretical description of the paraxial fluid of light and of its Bogoliubov excitations, we disregard the effect of photon losses by taking $\alpha=0$. This approach has the advantage of shining light on the general features without

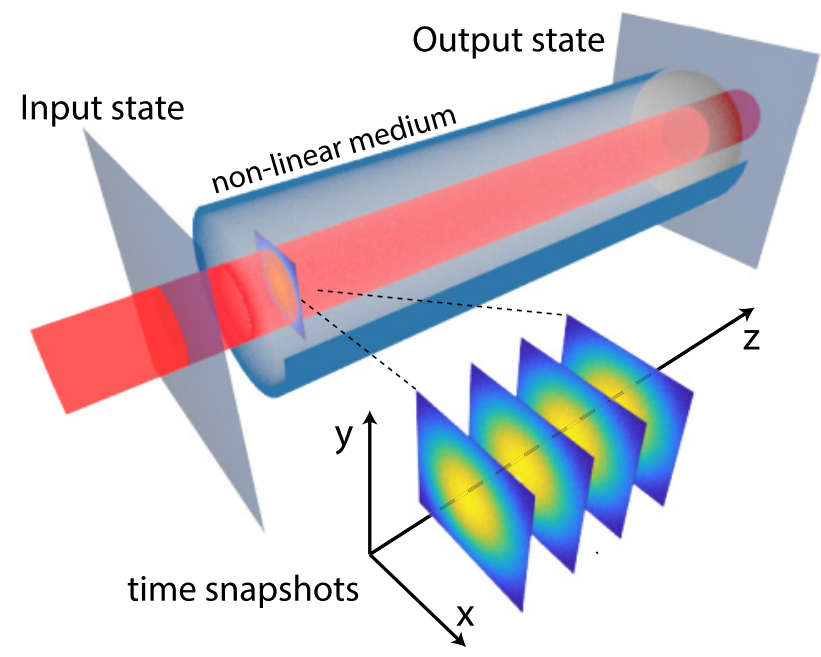

FIG. 1. Paraxial fluid of light. In the paraxial and scalar approximations, a laser beam propagates along the $z$ axis in a $\chi^{(3)}$ nonlinear medium according to the effective Gross-Pitaevskii equation (1). The field profile on each transverse $\mathbf{r}_{\perp}=(x, y)$ plane along the propagation direction $z$ is equivalent to a snapshot of the evolution of the paraxial fluid of light.

harming the generality of our conclusions. A complete theory including photon losses will be presented later in Fig. 5, showing no qualitative change.

In the ideal lossless case, we assume that the beam maintains a wide flat-top and $z$-independent intensity profile all along its propagation, so that the corresponding solution of (1) reads

$$
\mathcal{E}_{0}(z)=\sqrt{\rho_{0}} e^{-i k_{0} \Delta n z} .
$$

In this equation, $\rho_{0}$ is the density of the homogeneous paraxial fluid of light and $\Delta n=g \rho_{0} / k_{0}$ is the change of refractive index induced by the optical nonlinearity. A small departure from the uniform and stationary configuration (2) is described by a solution of (1) of the form

$$
\mathcal{E}\left(\mathbf{r}_{\perp}, z\right)=\mathcal{E}_{0}(z)+\delta \mathcal{E}\left(\mathbf{r}_{\perp}, z\right),
$$

where $\left|\delta \mathcal{E}\left(\mathbf{r}_{\perp}, z\right)\right| \ll\left|\mathcal{E}_{0}(z)\right|$. Such an expansion depicts weak-amplitude fluctuations (for example, intensity fluctuations) on top of the homogeneous (i.e., $\mathbf{r}_{\perp}$-independent) background defined in (2). The complex field $\delta \mathcal{E}\left(\mathbf{r}_{\perp}, z\right)$, which is a solution of the linearized version of (1), can be decomposed following the Bogoliubov approach [2] as a linear superposition of plane waves counterpropagating in the transverse $\mathbf{r}_{\perp}$ plane with opposite wave vectors $\pm \mathbf{k}_{\perp}$ and oscillating in the effective time $z$ at the same angular frequency $\Omega_{\mathrm{B}}\left(\mathbf{k}_{\perp}\right)$ :

$$
\begin{aligned}
\delta \mathcal{E}\left(\mathbf{r}_{\perp}, z\right)= & e^{-i k_{0} \Delta n z} \int \frac{d^{2} \mathbf{k}_{\perp}}{(2 \pi)^{2}}\left\{u\left(\mathbf{k}_{\perp}\right) b_{\mathbf{k}_{\perp}} e^{i\left[\mathbf{k}_{\perp} \cdot \mathbf{r}_{\perp}-\Omega_{\mathrm{B}}\left(\mathbf{k}_{\perp}\right) z\right]}\right. \\
& \left.+v^{*}\left(\mathbf{k}_{\perp}\right) b_{\mathbf{k}_{\perp}}^{*} e^{-i\left[\mathbf{k}_{\perp} \cdot \mathbf{r}_{\perp}-\Omega_{\mathrm{B}}\left(\mathbf{k}_{\perp}\right) z\right]}\right\} .
\end{aligned}
$$

In this expression, the complex amplitudes of the plane waves with wave vectors $\mathbf{k}_{\perp}$ and $-\mathbf{k}_{\perp}$ are, respectively, denoted by $u\left(\mathbf{k}_{\perp}\right) b_{\mathbf{k}_{\perp}}$ and $v^{*}\left(\mathbf{k}_{\perp}\right) b_{\mathbf{k}_{\perp}}^{*}$, where $b_{\mathbf{k}_{\perp}}$ is chosen to be homogeneous to a voltage times a length so that $u\left(\mathbf{k}_{\perp}\right)$ and 
$v\left(\mathbf{k}_{\perp}\right)$ are by construction dimensionless. The latter satisfy the eigenvalue problem [2]

$$
\begin{aligned}
& \mathcal{L}\left(\mathbf{k}_{\perp}\right)\left[\begin{array}{c}
u\left(\mathbf{k}_{\perp}\right) \\
v\left(\mathbf{k}_{\perp}\right)
\end{array}\right]=\Omega_{\mathrm{B}}\left(\mathbf{k}_{\perp}\right)\left[\begin{array}{c}
u\left(\mathbf{k}_{\perp}\right) \\
v\left(\mathbf{k}_{\perp}\right)
\end{array}\right], \quad \text { where } \\
& \mathcal{L}\left(\mathbf{k}_{\perp}\right)=\left[\begin{array}{cc}
k_{\perp}^{2} /\left(2 k_{0}\right)+k_{0} \Delta n & k_{0} \Delta n \\
-k_{0} \Delta n & -k_{\perp}^{2} /\left(2 k_{0}\right)-k_{0} \Delta n
\end{array}\right],
\end{aligned}
$$

with the wave number $k_{\perp}=\left|\mathbf{k}_{\perp}\right|$. Without loss of generality, we take $u\left(\mathbf{k}_{\perp}\right)$ and $v\left(\mathbf{k}_{\perp}\right)$ to be real. Setting the normalization condition $u^{2}\left(\mathbf{k}_{\perp}\right)-v^{2}\left(\mathbf{k}_{\perp}\right)=1$, we get the dispersion relation

$$
\begin{aligned}
& \Omega_{\mathrm{B}}\left(\mathbf{k}_{\perp}\right)=\sqrt{\frac{k_{\perp}^{2}}{2 k_{0}}\left(\frac{k_{\perp}^{2}}{2 k_{0}}+2 k_{0} \Delta n\right)} \text { and } \\
& u\left(\mathbf{k}_{\perp}\right) \pm v\left(\mathbf{k}_{\perp}\right)=\left[\frac{k_{\perp}^{2}}{2 k_{0}} / \Omega_{\mathrm{B}}\left(\mathbf{k}_{\perp}\right)\right]^{ \pm \frac{1}{2}} .
\end{aligned}
$$

Equation (6) is the optical analog of the Bogoliubov excitation spectrum of a homogeneous two-dimensional atomic BEC at rest and (7) gives the $\mathbf{k}_{\perp}$ dependence of the Bogoliubov amplitudes $u\left(\mathbf{k}_{\perp}\right)$ and $v\left(\mathbf{k}_{\perp}\right)$. Here, the linear combinations $u+v$ and $u-v$, respectively, correspond to the density and phase amplitudes of the Bogoliubov collective wave in wave-vector space.

From (6), we can extract the peculiar behavior of the Bogoliubov dispersion relation $\Omega_{\mathrm{B}}\left(\mathbf{k}_{\perp}\right)$, which is linear (soundlike) at small $\mathbf{k}_{\perp}$ and parabolic (free-particle-like) at large $\mathbf{k}_{\perp}$ :

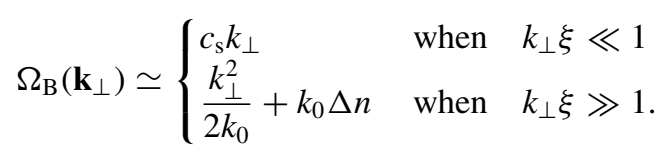

These asymptotic behaviors bring up the optical analogs of the Bogoliubov sound velocity, $c_{\mathrm{s}}=\sqrt{\Delta n}$, and of the healing length, $\xi=1 /\left(k_{0} \sqrt{\Delta n}\right)=1 /\left(k_{0} c_{\mathrm{s}}\right)$, of atomic BECs. In the present optical context, $c_{\mathrm{s}}$ is by construction dimensionless, as it corresponds to the propagation angle with respect to the $z$ axis. The peculiar refraction properties corresponding to the constant $c_{s}$ in the $k \xi \rightarrow 0$ limit were highlighted in Ref. [16]. In the large $k \xi$ limit, the shift in $\Omega_{B}\left(\mathbf{k}_{\perp}\right)$ is simply linked to the nonlinear refractive index change.

\section{EXTRACTING THE BOGOLIUBOV DISPERSION RELATION FROM THE PHASE VELOCITY}

The phase velocity $v_{\mathrm{ph}}\left(\mathbf{k}_{\perp}\right)$ of a Bogoliubov plane wave with wave vector $\mathbf{k}_{\perp}$ is related to the Bogoliubov dispersion relation $\Omega_{\mathrm{B}}\left(\mathbf{k}_{\perp}\right)$ through

$$
v_{\mathrm{ph}}\left(\mathbf{k}_{\perp}\right)=\frac{\Omega_{\mathrm{B}}\left(\mathbf{k}_{\perp}\right)}{k_{\perp}} .
$$

Therefore, it is expected that we can directly reconstruct $\Omega_{\mathrm{B}}\left(\mathbf{k}_{\perp}\right)$ from the measurement of $v_{\mathrm{ph}}\left(\mathbf{k}_{\perp}\right)$, which can be assessed from the measurement of the distance

$$
S\left(\mathbf{k}_{\perp}\right)=v_{\mathrm{ph}}\left(\mathbf{k}_{\perp}\right) L
$$

that the Bogoliubov excitation travels in the transverse plane between the effective times $z=0$ and $z=L$, where $L$ stands for the length of the nonlinear medium.
In the experimental configuration initially proposed in Ref. [15] and studied here, $S\left(\mathbf{k}_{\perp}\right)$ corresponds to the transverse displacement of a weak interference pattern obtained by overlapping a large-intensity flat-top background with a low-intensity probe, slightly tilted by an angle $\theta_{\mathrm{i}}$ with the $z$ axis along which the background propagates. These two beams come from the same laser, have the same frequency and polarization, and thus interfere, producing a small fluctuation $\delta \mathcal{E}\left(\mathbf{r}_{\perp}, z\right)$ on top of the background envelope $\mathcal{E}_{0}(z)$ in the nonlinear medium. The norm $k_{\perp}=\left(k_{0} / n\right) \sin \theta_{\mathrm{i}}$ of the transverse wave vector of the incident probe is controlled by changing $\theta_{\mathrm{i}}$, which must be small enough so that the whole optical system falls into the paraxial limit $k_{\perp} \ll k_{0}$ considered here.

After propagation inside the medium of length $L$, the background ("bg") and the probe ("p") have accumulated different phases $\Phi_{\text {bg }}$ and $\Phi_{\mathrm{p}}\left(\mathbf{k}_{\perp}\right)$. According to (4), the latter depends on the Bogoliubov dispersion relation $\Omega_{\mathrm{B}}\left(\mathbf{k}_{\perp}\right)$. The difference

$$
\Phi\left(\mathbf{k}_{\perp}\right)=\Phi_{\mathrm{p}}\left(\mathbf{k}_{\perp}\right)-\Phi_{\mathrm{bg}}
$$

between these two phases is responsible for an interference pattern in the transverse plane, shifted by

$$
S\left(\mathbf{k}_{\perp}\right)=\frac{\Phi\left(\mathbf{k}_{\perp}\right)}{k_{\perp}} .
$$

Experimentally, it is possible to have access to

$$
\Delta S\left(\mathbf{k}_{\perp}\right)=S_{\mathrm{NL}}\left(\mathbf{k}_{\perp}\right)-S_{\mathrm{L}}\left(\mathbf{k}_{\perp}\right),
$$

the relative deviation between the fringes patterns obtained at high and low background intensity, that is, in the nonlinear ("NL") regime and the linear ("L") one, respectively. This quantity can be, at first, estimated in a geometrical approach, as detailed below.

\section{A. Geometrical approach}

In the linear regime, simple geometry yields the following expressions for the phases accumulated by the background and the probe beams:

$$
\begin{gathered}
\Phi_{\mathrm{bg}, \mathrm{L}}=k_{0} L \text { and } \\
\Phi_{\mathrm{p}, \mathrm{L}}\left(\mathbf{k}_{\perp}\right)=k_{0} \sqrt{L^{2}+L^{2} \tan ^{2} \theta_{\mathrm{r}}} \simeq k_{0} L\left(1+\frac{\theta_{\mathrm{r}}^{2}}{2}\right),
\end{gathered}
$$

where $\theta_{\mathrm{r}} \simeq \theta_{i} / n$ is the refraction angle of the probe at the entrance of the medium. Using $k_{\perp} \simeq\left(k_{0} / n\right) \theta_{\mathrm{i}}$, we then obtain

$$
\Phi_{\mathrm{L}}\left(\mathbf{k}_{\perp}\right)=\frac{k_{\perp}^{2}}{2 k_{0}} L .
$$

In a geometrical approach, the same formula is supposed to hold in the nonlinear regime provided the free-particle dispersion relation $k_{\perp}^{2} /\left(2 k_{0}\right)$ is replaced with the Bogoliubov spectrum (6) and we obtain using (11)

$$
\Phi_{\mathrm{NL}}\left(\mathbf{k}_{\perp}\right)=\Omega_{\mathrm{B}}\left(\mathbf{k}_{\perp}\right) L .
$$

In the light of Eqs. (12) and (13), this geometric approach then leads to

$$
\Delta S\left(\mathbf{k}_{\perp}\right)=\frac{k_{\perp}}{2 k_{0}}\left[\sqrt{1+\Delta n\left(\frac{2 k_{0}}{k_{\perp}}\right)^{2}}-1\right] L .
$$


This expression states that $\Delta S\left(\mathbf{k}_{\perp}\right)$ saturates to a constant value proportional to the Bogoliubov speed of sound in the deep phonon regime:

$$
\Delta S\left(\mathbf{k}_{\perp}\right) \underset{k_{\perp}}{\simeq} \underset{\xi \ll 1}{\simeq} \sqrt{\Delta n} L=c_{\mathrm{S}} L .
$$

This approach has been experimentally implemented for nonlocal photon fluids [15]. In particular (19) suggests that the displacement $\Delta S\left(\mathbf{k}_{\perp}\right)$ tends at small $k_{\perp}$ towards the intuitive geometric value given by the product of the sound velocity $c_{S}$ by the effective time $L$.

Surprisingly, this geometric approach differs drastically from the results of the full theory in Refs. $[17,18]$, which predict instead a linear increase of $\Delta S\left(\mathbf{k}_{\perp}\right)$ with $\Lambda=2 \pi / k_{\perp}$ at small $k_{\perp}$, even in the limit of weak interactions $(\Delta n \rightarrow 0)$. In the following, we explain the physical origin of this correction and show that interferences between the counterpropagating Bogoliubov collective excitations are responsible for the disagreement between Ref. [15] and Refs. [17,18] in the sonic regime $\left(k_{\perp} \xi \ll 1\right)$.

\section{B. Theoretical model including the interferences between counterpropagating Bogoliubov excitations}

Let us first introduce qualitatively this effect before deriving the full analytical solution. When the background and the probe enter the nonlinear medium, both experience a sudden jump of the $\chi^{(3)}$ susceptibility, analogous to a quantum quench of the interactions [24-26]. This generates a conjugate beam, due to the boundary condition on the electric-field amplitude at the interface. The conjugate field oscillates at the same frequency as the background and the probe, and propagates in the transverse direction with a wave vector $-\mathbf{k}_{\perp}$ opposite to the one of the incident probe. In optics, this thirdorder nonlinear wave-mixing process is known as degenerate four-wave mixing [27-29].

Interestingly, the interferences between the two counterpropagating Bogoliubov excitations (the probe and the conjugate within the medium), neglected in the geometrical model [15], are continuously taking place all along their propagation in the nonlinear medium. Since the pump, probe, and conjugate have the same frequency, they do fulfill the phase-matching condition only when they are copropagating, that is, when $k_{\perp}=0$. This can be seen by evaluating the ratio of the conjugate Bogoliubov amplitude $v\left(\mathbf{k}_{\perp}\right)$ to the probe one $u\left(\mathbf{k}_{\perp}\right)$ using (7). In the free-particle regime $k_{\perp} \xi \gg 1$, this ratio is small as it scales as $1 /\left(k_{\perp}^{2} \xi^{2}\right)$. In this limit, the impact of the interferences between the conjugate and the probe can be safely neglected and (17) is valid, as shown in the next section. However, in the phonon regime $k_{\perp} \xi \ll 1,\left|v\left(\mathbf{k}_{\perp}\right) / u\left(\mathbf{k}_{\perp}\right)\right| \simeq 1$ and a full model taking into account the interferences between the counterpropagating Bogoliubov excitations gives drastically different results from the geometric approach detailed above.

In the case of a finite diameter probe mode (not a plane wave), the geometric model of (18) is recovered when the length $L$ of the medium is long enough for the probe and conjugate wave packets to get spatially separated during the propagation [8]. In this limit, the distance between of the wave-packet centers gives access to the group velocity [16], while the position of the fringes within the wave packets gives access to the phase velocity. For realistic parameters, this requires impractically long samples.

In the following, we derive an exact expression for the relative phase $\Phi_{\mathrm{NL}}\left(\mathbf{k}_{\perp}\right)=\Phi_{\mathrm{p}, \mathrm{NL}}\left(\mathbf{k}_{\perp}\right)-\Phi_{\mathrm{bg}, \mathrm{NL}}$ accumulated by the probe with respect to the background after propagation through the medium. We use an approach similar to the quantum optics input-output formalism [30,31] with a description of the medium given by the Bogoliubov theory [17].

In air (i.e., $z<0$ and $z>L$ ) the envelope of the electric field including the background and its fluctuations may be expanded as

$\mathcal{E}_{\text {air }}\left(\mathbf{r}_{\perp}, z\right)=\sqrt{\rho_{\text {air }}(z)} e^{i \Phi_{\text {air }}(z)}+e^{i \Phi_{\text {air }}(z)} \int \frac{d^{2} \mathbf{k}_{\perp}}{(2 \pi)^{2}} a_{\mathbf{k}_{\perp}}(z) e^{i \mathbf{k}_{\perp} \cdot \mathbf{r}_{\perp}}$.

In this equation, $\rho_{\text {air }}(z)$ and $\Phi_{\text {air }}(z)$ are the density and the phase of the homogeneous background in air. Due to the conservation of energy at $z=0$ and $z=L$, the densities are related by $\rho_{\text {air }}=\rho_{\text {air }}(z>L)=n \rho_{0}$, while the phases are $\Phi_{\text {air }}(z<0)=0$ and $\Phi_{\text {air }}(z>L)=-k_{0} \Delta n L$. In $(20), a_{\mathbf{k}_{\perp}}(z)$ denotes the Fourier amplitude of the fluctuations superimposed on the background in air. In our experiment, only one $\mathbf{k}_{\perp}$ component (corresponding to the probe mode for $z=0^{-}$) is injected into the medium and all the other modes are set to zero. Using the sign convention adopted in (4), the phase difference $\Phi_{\mathrm{NL}}\left(\mathbf{k}_{\perp}\right)$ can then be expressed as follows:

$$
\Phi_{\mathrm{NL}}\left(\mathbf{k}_{\perp}\right)=-\arg \left[\frac{a_{\mathbf{k}_{\perp}}\left(L^{+}\right)}{a_{\mathbf{k}_{\perp}}\left(0^{-}\right)}\right] .
$$

To derive the input-output relation between $a_{\mathbf{k}_{\perp}}\left(L^{+}\right)$and $a_{\mathbf{k}_{\perp}}\left(0^{-}\right)$we need to use both energy conservation at the interfaces and the Bogoliubov formalism for the evolution within the medium. In a first step, when entering the medium at $z=0$, the probe of amplitude $a_{\mathbf{k}_{\perp}}\left(0^{-}\right)$transforms, by energy conservation, into $a_{\mathbf{k}_{\perp}}\left(0^{+}\right)=\sqrt{n} a_{\mathbf{k}_{\perp}}\left(0^{-}\right)$. Then, in analogy to the quantum formalism of dilute Bose gases, we can consider the term $a_{\mathbf{k}_{\perp}}\left(0^{+}\right)$to be equivalent to the annihilation operator for the weakly interacting particles. Following the Bogoliubov approach, it can be connected to the noninteracting Bogoliubov operators $b_{\mathbf{k}_{\perp}}$ using the transformation

$$
\left[\begin{array}{c}
a_{\mathbf{k}_{\perp}}\left(0^{+}\right) \\
a_{-\mathbf{k}_{\perp}^{*}}^{*}\left(0^{+}\right)
\end{array}\right]=\left[\begin{array}{cc}
u\left(\mathbf{k}_{\perp}\right) & v\left(\mathbf{k}_{\perp}\right) \\
v\left(\mathbf{k}_{\perp}\right) & u\left(\mathbf{k}_{\perp}\right)
\end{array}\right]\left[\begin{array}{c}
b_{\mathbf{k}_{\perp}} \\
b_{-\mathbf{k}_{\perp}}^{*}
\end{array}\right] .
$$

Thereafter, the counterpropagating Bogoliubov excitations evolve along the optical axis, accumulating a propagation phase provided by the Bogoliubov dispersion relation $\Omega_{\mathrm{B}}\left(\mathbf{k}_{\perp}\right)$. This evolution is analogous to those observed after a quench in atomic BEC, and leads to synchronized phases between the counterpropagating phonon modes $\left(b_{\mathbf{k}_{\perp}}\right.$ and $\left.b_{-\mathbf{k}_{\perp}}^{*}\right)$. Interestingly, this synchronized effect is at the origin of Sakharov oscillations [21]. We obtain

$$
\left[\begin{array}{c}
a_{\mathbf{k}_{\perp}}\left(L^{-}\right) \\
a_{-\mathbf{k}_{\perp}^{*}}^{*}\left(L^{-}\right)
\end{array}\right]=\left[\begin{array}{cc}
u\left(\mathbf{k}_{\perp}\right) e^{-i \Omega_{\mathrm{B}}\left(\mathbf{k}_{\perp}\right) L} & v\left(\mathbf{k}_{\perp}\right) e^{i \Omega_{\mathrm{B}}\left(\mathbf{k}_{\perp}\right) L} \\
v\left(\mathbf{k}_{\perp}\right) e^{-i \Omega_{\mathrm{B}}\left(\mathbf{k}_{\perp}\right) L} & u\left(\mathbf{k}_{\perp}\right) e^{i \Omega_{\mathrm{B}}\left(\mathbf{k}_{\perp}\right) L}
\end{array}\right]\left[\begin{array}{c}
b_{\mathbf{k}_{\perp}} \\
b_{-\mathbf{k}_{\perp}}^{*}
\end{array}\right] .
$$

We can then invert (22) using the Bogoliubov normalization $u^{2}\left(\mathbf{k}_{\perp}\right)-v^{2}\left(\mathbf{k}_{\perp}\right)=1$ and inject the result into the right-hand side of (23). Finally, taking into account energy conservation 
at the medium output $a_{\mathbf{k}_{\perp}}\left(L^{-}\right)=\sqrt{n} a_{\mathbf{k}_{\perp}}\left(L^{+}\right)$, we obtain the following input-output relations:

$$
\left[\begin{array}{c}
a_{\mathbf{k}_{\perp}}\left(L^{+}\right) \\
a_{-\mathbf{k}_{\perp}}^{*}\left(L^{+}\right)
\end{array}\right]=\left[\begin{array}{ll}
U\left(\mathbf{k}_{\perp}\right) & V^{*}\left(-\mathbf{k}_{\perp}\right) \\
V\left(\mathbf{k}_{\perp}\right) & U^{*}\left(-\mathbf{k}_{\perp}\right)
\end{array}\right]\left[\begin{array}{c}
a_{\mathbf{k}_{\perp}}\left(0^{-}\right) \\
a_{-\mathbf{k}_{\perp}}^{*}\left(0^{-}\right)
\end{array}\right],
$$

where $U\left(\mathbf{k}_{\perp}\right)$ and $V\left(\mathbf{k}_{\perp}\right)$ are defined by

$$
\begin{gathered}
U\left(\mathbf{k}_{\perp}\right)=u^{2}\left(\mathbf{k}_{\perp}\right) e^{-i \Omega_{\mathrm{B}}\left(\mathbf{k}_{\perp}\right) L}-v^{2}\left(\mathbf{k}_{\perp}\right) e^{i \Omega_{\mathrm{B}}\left(\mathbf{k}_{\perp}\right) L} \quad \text { and } \\
V\left(\mathbf{k}_{\perp}\right)=-2 i u\left(\mathbf{k}_{\perp}\right) v\left(\mathbf{k}_{\perp}\right) \sin \left[\Omega_{\mathrm{B}}\left(\mathbf{k}_{\perp}\right) L\right] .
\end{gathered}
$$

In our configuration, right before the medium entrance $(z=$ $0^{-}$), the mode with wave vector $-\mathbf{k}_{\perp}$ has a zero amplitude (i.e., the conjugate mode is seeded by vacuum) and therefore we set $a_{-\mathbf{k}_{\perp}}^{*}\left(0^{-}\right)=0$ in (24). As a result, we eventually come to the simple relation

$$
a_{\mathbf{k}_{\perp}}\left(L^{+}\right)=U\left(\mathbf{k}_{\perp}\right) a_{\mathbf{k}_{\perp}}\left(0^{-}\right),
$$

from which we can simplify (21) and obtain the $\Omega_{\mathrm{B}}\left(\mathbf{k}_{\perp}\right)$ dependence of $\Phi_{\mathrm{NL}}\left(\mathbf{k}_{\perp}\right)$ from Eqs. (6), (7), and (25):

$$
\begin{aligned}
\Phi_{\mathrm{NL}}\left(\mathbf{k}_{\perp}\right) & =-\arg \left[U\left(\mathbf{k}_{\perp}\right)\right] \\
& =\Omega_{B}\left(\mathbf{k}_{\perp}\right) L-\arg \left[u^{2}\left(\mathbf{k}_{\perp}\right)-v^{2}\left(\mathbf{k}_{\perp}\right) e^{2 i \Omega_{B}\left(\mathbf{k}_{\perp}\right) L}\right] \\
& =\arctan \left\{\frac{\left[k_{\perp}^{2} /\left(2 k_{0}\right)\right]^{2}+\Omega_{\mathrm{B}}\left(\mathbf{k}_{\perp}\right)^{2}}{k_{\perp}^{2} / k_{0} \times \Omega_{\mathrm{B}}\left(\mathbf{k}_{\perp}\right)} \tan \left[\Omega_{\mathrm{B}}\left(\mathbf{k}_{\perp}\right) L\right]\right\} .
\end{aligned}
$$

The second expression of (28) allows for a direct understanding of the role of the interferences between Bogoliubov phonon excitations in the correction to (18).

In the free-particle regime $\left(k_{\perp} \xi \gg 1\right)$, the $v^{2}$ term in the second expression of (28) is negligible. This is equivalent to saying that the phase-matching condition is not fulfilled and the four-wave-mixing process is inefficient to create the conjugate mode. Since $u\left(\mathbf{k}_{\perp}\right)$ is real, we get

$$
\Phi_{\mathrm{NL}}\left(\mathbf{k}_{\perp}\right) \underset{k_{\perp}}{\sim} \underset{\xi}{\widetilde{\xi}} \Omega_{\mathrm{B}}\left(\mathbf{k}_{\perp}\right) L .
$$

This limit exactly corresponds to the geometric model of (17) and correctly describes the transverse fringes displacement $\Delta S\left(\mathbf{k}_{\perp}\right)$. However, this approximation is only valid in the parabolic dispersion limit at large momenta $k_{\perp} \xi \gg 1$.

In the phonon regime $\left(k_{\perp} \xi \ll 1\right)$ where superfluidity is manifest, there are fundamental differences between the predictions of the geometrical approach and the full model, because the $v^{2}$ term cannot be neglected anymore in the second expression of (28). This interference term leads to a correction to (17), which we can expand analytically in the limit $k_{\perp} \xi \ll 1$ to get at the leading order

$$
\Phi_{\mathrm{NL}}\left(\mathbf{k}_{\perp}\right) \underset{k_{\perp} \xi \ll 1}{=} \arctan \left(2 k_{0} \Delta n L\right)+O\left(k_{\perp}^{2} \xi^{2}\right) .
$$

An essential feature of (30) is that the nonlinear phase difference $\Phi_{\mathrm{NL}}\left(\mathbf{k}_{\perp}\right)$ at the medium output converges towards a constant nonzero value for small $\mathbf{k}_{\perp}$. This can be clearly seen in Fig. 2(a) for realistic experimental parameters. This nonzero value holds independently of the strength of the interactions $\Delta n$, and therefore is a general feature of paraxial fluids of light and a direct consequence of the interferences between Bogoliubov phonons. At large $\Delta n$, this offset saturates towards $\pi / 2$.
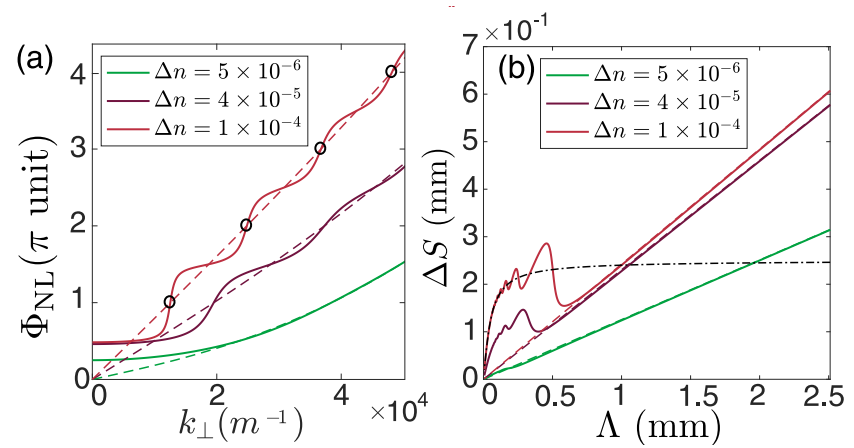

FIG. 2. (a) Nonlinear phase shift $\Phi_{\mathrm{NL}}\left(\mathbf{k}_{\perp}\right)$ and (b) relative fringes displacement $\Delta S(\Lambda)$ as functions of, respectively, the Bogoliubov wave number $k_{\perp}=\left|\mathbf{k}_{\perp}\right|$ and the Bogoliubov wavelength $\Lambda=2 \pi / k_{\perp}$ for different values of the optical nonlinearity $\Delta n$. (a) The phase shift $\Phi_{\mathrm{NL}}\left(\mathbf{k}_{\perp}\right)$ generally follows $\Omega_{\mathrm{B}}\left(\mathbf{k}_{\perp}\right) L$ on average (dashed lines), except for small $k_{\perp}$, where it saturates at a nonzero value for all values of $\Delta n \neq 0$. According to (30), the $k_{\perp}=0$ limit $\Phi_{\mathrm{NL}}\left(\mathbf{k}_{\perp}=0\right)$ is a growing function of $\Delta n$ and tends towards $\pi / 2$ for large $\Delta n$. (b) The staircase structure of $\Phi_{\mathrm{NL}}\left(\mathbf{k}_{\perp}\right)$ translates into oscillations in $\Delta S(\Lambda)$, most visible for large interactions. At long $\Lambda, \Delta S(\Lambda)$ increases linearly according to (31). This trend is present even for weak interactions $(\Delta n \rightarrow 0)$. Solid lines correspond to the full model and the black dash-dotted line shows for comparison the displacement obtained from the geometric approach of (18) with $\Delta n=10^{-4}$.

In between these two asymptotic limits, we also observe numerically a smooth staircase structure, which follows on average the trend of the geometric prediction (18) in the large$k_{\perp}$ limit [dashed lines in Fig. 2(a)]. This staircase structure becomes more and more visible as the optical nonlinearity $\Delta n$ increases. This effect is less robust than the nonzero value of $\Phi_{\mathrm{NL}}\left(\mathbf{k}_{\perp}\right)$ in the small $k_{\perp}$ limit previously described and does not hold for weak interactions $\Delta n$ [see the green curve of Fig. 2(a)]. Therefore, in order to evidence the presence of interferences between the Bogoliubov phonons, we will focus our attention on the phase difference at small $k_{\perp}$ by looking at the displacement $\Delta S$ as a function of $\Lambda=2 \pi / k_{\perp}$.

In Fig. 2(b), we present this displacement $\Delta S$ (accessible experimentally) as a function of the density modulation wavelength $\Lambda$. Because of the staircase structure of $\Phi_{\mathrm{NL}}\left(\mathbf{k}_{\perp}\right)$, the displacement $\Delta S(\Lambda)$ oscillates at short $\Lambda$. Once again this effect disappears for weak interactions $\Delta n$ [green curve of Fig. 2(b)]. In contrast, the linear increase of $\Delta S(\Lambda)$ when $\Lambda \gg \xi$ is always present for all $\Delta n$ and can be computed from (30) as

$$
\Delta S(\Lambda) \underset{\Lambda / \xi \gg 1}{\sim} \frac{\arctan \left(2 k_{0} \Delta n L\right)}{2 \pi} \Lambda .
$$

This expression significantly contrasts with (19), obtained within the geometrical approach. For comparison, the total displacement [Eq. (18)] predicted by the geometric method is plotted for $\Delta n=10^{-4}$ in black dash-dotted line in Fig. 2(b). As expected, the two descriptions match in the free-particle regime, but the linear increase [Eq. (31)] at long $\Lambda$ is only present in the full model and not predicted by (18).

In the next section, we explore experimentally this configuration in a hot atomic vapor to compare and verify the 


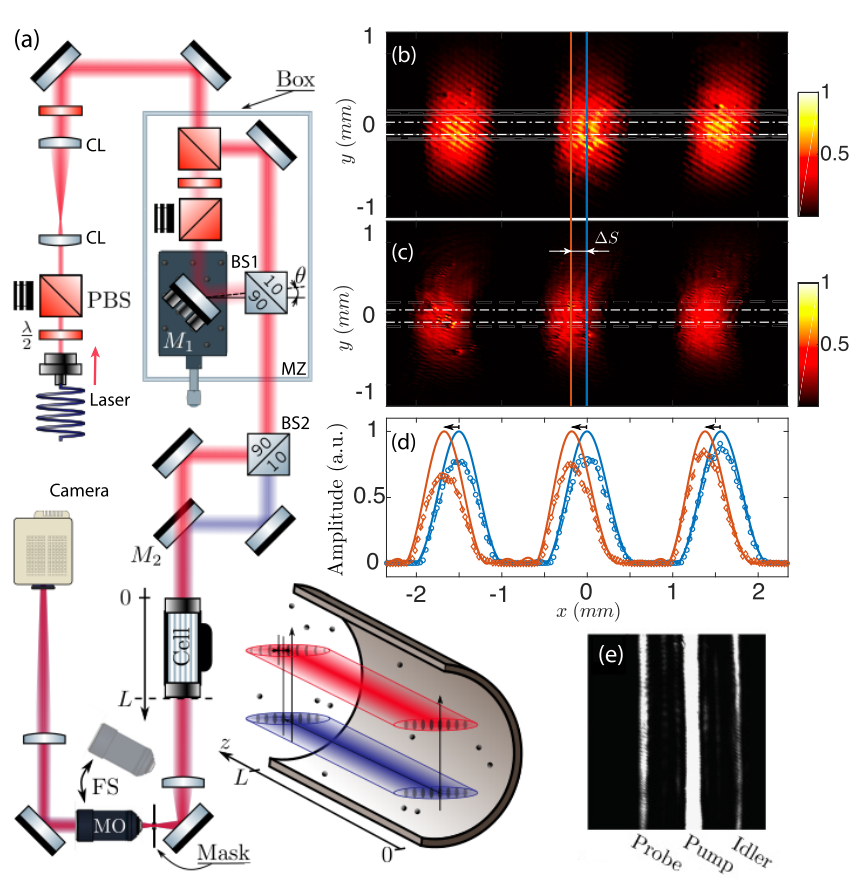

FIG. 3. (a) Experimental setup. A laser is shaped with two cylindrical lenses (CL). It is split and recombined (BS1) within an unbalanced Mach-Zehnder (MZ) interferometer to create a low contrast fringes pattern. Two sets of fringes (low and high intensity) are vertically shifted using a 90:10 beam-splitter (BS2) before going into an atomic vapor cell. The cell output is imaged on a camera after filtering in the Fourier space (FS). (b), (c) Background-subtracted images reveal the small amplitude density modulation which propagates on a low (b) and a high intensity background fluid (c). The blue and red points in (d) are obtained by integrating the intensity in between the white dashed lines in (b) and (c), respectively. We first filter out the high-frequency noise (dashed lines) and then normalize the envelopes (solid lines). The shift is computed by measuring the nearest peak-to-peak distance between the solid lines. (e) Fourier space image obtained by inserting a microscope objective (MO).

predictions of the two models. We will show evidences of the interference between the counterpropagating Bogoliubov collective excitations at small $k_{\perp}$ and of their role on the measurement of the dispersion relation following (31).

\section{EXPERIMENTAL EVIDENCES OF INTERFERENCES BETWEEN BOGOLIUBOV EXCITATIONS}

\section{A. Experimental setup}

Our experimental setup is sketched in Fig. 3(a). A continuous-wave laser field at $780 \mathrm{~nm}$ is elongated in the $x$ direction using a set of two cylindrical lenses. This cylindrical telescope is slightly defocused in order to loosely focus the beam onto the medium input facet. In this plane, the minor axis width $\omega_{0, y}$ (radius at $1 / e^{2}$ ) is $500 \mu \mathrm{m}$ while the major axis one $\omega_{0, x}$ is $1 \mathrm{~cm}$. The Rayleigh length associated to $\omega_{0, y}$ is much longer than the cell length $(L=7.5 \mathrm{~cm})$. The cell is filled with an isotopically pure ${ }^{85} \mathrm{Rb}$ vapor heated up to $400 \mathrm{~K}$. The laser frequency is $2.6-\mathrm{GHz}$ red-detuned with respect to the $F=3 \rightarrow F^{\prime}$ transition of the ${ }^{85} \mathrm{Rb} D_{2}$ line, which ensures a linear index of refraction close to 1 and a transmission larger than $60 \%$.

The weak intensity modulation pattern is created using an unbalanced Mach-Zehnder interferometer. The beam is then split in two with a 90:10 $(R: T)$ beam splitter and recombined with a vertical shift to have simultaneously a weak intensity modulation evolving on top of a high intensity beam forming the photon fluid. The medium exit plane is imaged on a camera with a $4 f$ telescope. By inserting a microscope objective on the beam path, we can image the momentum distribution [Fig. 3(e)]. Spatial Fourier filtering using a razor blade is conducted in this plane to filter out the conjugate beam that blurs the fringes pattern. As sketched in Fig. 3, we perform simultaneously the experiment in two regimes: (i) low fluid density and (ii) high fluid density. The low density fluid corresponds to the case of a negligible nonlinearity and provides a reference $\left(\Phi_{\mathrm{L}}\right)$ for the fringe displacement. Comparing both patterns we observe the fringe displacement and measure $\Delta S$.

\section{B. Data analysis and results}

After removing the background intensity distribution to keep only the small density modulation on top of it, typical interference patterns obtained at the medium output plane are shown in Figs. 3(b) and 3(c). The displacement between the fringes of the low intensity reference [Fig. 3(b)] and high intensity fluid [Fig. 3(c)] $\Delta S$ is clearly visible. We can note that the fringes are slightly bent in Fig. 3(c) because the intensity profile along the vertical axis is Gaussian and therefore the nonlinear phase shift accumulated during the propagation depends on $y$. In order to avoid errors during the data analysis, we average the intensity profile over the central region in between the white dotted line in Figs. 3(b) and 3(c).

After averaging, the resulting profiles are plotted in Fig. 3(d): the blue points are for the low intensity reference [Fig. 3(a)] while the red ones are for the high intensity nonlinear case [Fig. 3(b)]. The high-frequency noise is filtered out and we remove the envelopes using a cubic spline interpolation method to normalize it and obtain the blue and red solid curves. The relative displacement is computed by averaging on several fringes the distance (black arrows) between the nearest maxima in the low intensity reference and in the high intensity case.

In Fig. 4(a), we present the experimentally measured $\Delta S$ as a function of the modulation wavelength $\Lambda$. The probe power is taken to ensure a modulation depth of less than $5 \%$. The full model is shown in blue solid line. For comparison, the geometrical model computed with (18) is plotted in black dash-dotted line. These experimental results are a clear evidence that the geometrical model fails to describe the displacement $\Delta S$ at large $\Lambda$. Indeed, at large $\Lambda$, we observe a clear signature of the linear increase of $\Delta S$, as predicted by the full model. By including the interferences between elementary Bogoliubov excitations, the full model also allows us to predict the value of the slope as a function of the nonlinear refractive index change $\Delta n$. To verify the consistency of our model, we repeated the measurement of $\Delta S$ for various field intensities $I$ and estimated $\Delta n$ from the theoretical predictions, using (31). An intriguing feature of this equation is the nonlinear behavior of the phase shift and the saturation at large 

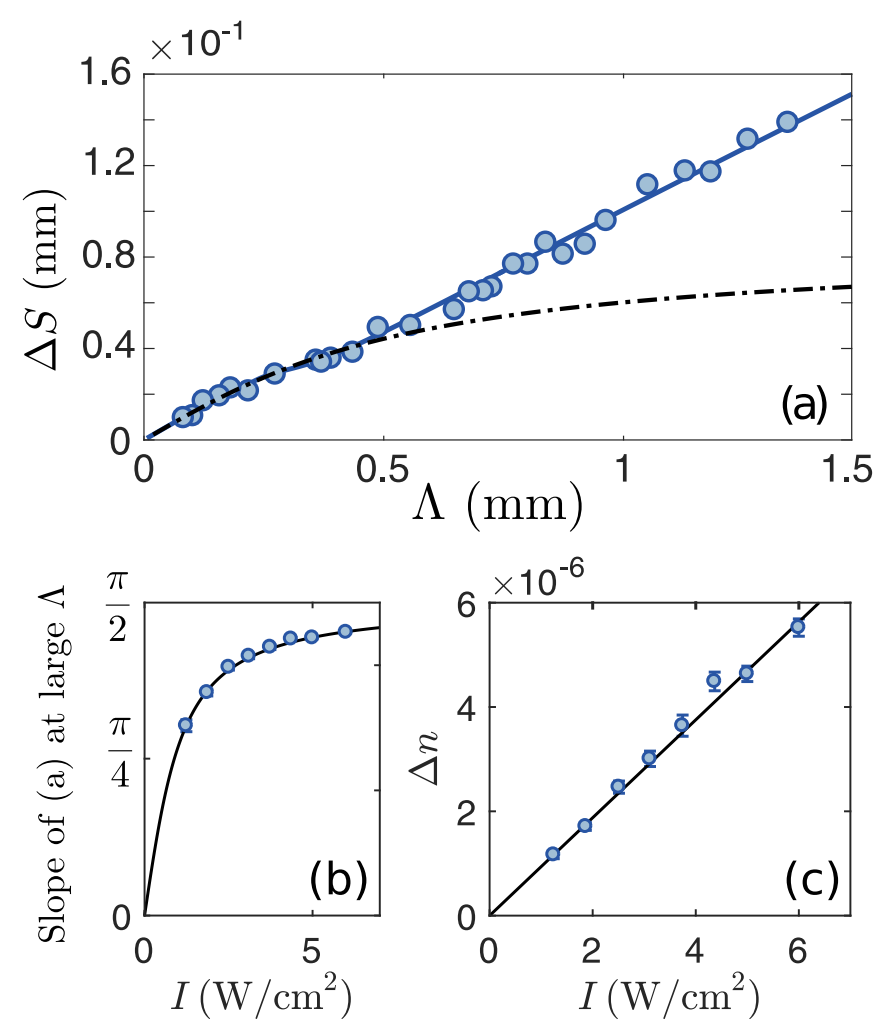

FIG. 4. (a) Displacement $\Delta S$ as a function of the modulation wavelength $\Lambda$ for a fluid intensity of $1.3 \mathrm{~W} \mathrm{~cm}^{-2}$. The laser is 2.6-GHz red-detuned with respect to the $F=3 \rightarrow F^{\prime}$ transition of the ${ }^{85} \mathrm{Rb} D_{2}$ line and the cell length is $7.5 \mathrm{~cm}$. The experimental data (blue circle) are fitted with the full theory (blue line) for $\Delta n=$ $1.3 \times 10^{-6}$. For comparison, the displacement obtained using (18) has been plotted (black dash-dotted line). (b) Slope of the asymptotic linear increase of $\Delta S$ at large $\Lambda$ as a function of the fluid intensity. (c) $\Delta n$ extracted with Eq. (30) from the slope of $\Delta S$ at large $\Lambda$ as a function of the fluid intensity plotted in (b). The linear scaling of $\Delta n$ with $I$ confirms that we are not saturating the nonlinearity, i.e., $\Delta n=n_{2} I$ with $n_{2}=1 \times 10^{-10} \mathrm{~m}^{2} / \mathrm{W}$.

interaction $\Delta n$ [Fig. 4(b)]. However, as visible in Fig. 4(c), the value of $\Delta n$ extracted from (31) depends linearly with the background intensity $I$ as expected for a Kerr medium and it validates our experimental approach.

\section{INTERFERENCES BETWEEN BOGOLIUBOV WAVES}

\section{A. Is this interference effect robust with respect to corrections to the lossless local Kerr model?}

Several nonlinear media have been proposed and implemented for fluid of light experiments, including atomic vapor [14,32], methanol [12], photorefractive crystal [10,11,33], and nematic liquid crystals [18]. In these systems the microscopic origin of light-matter interaction strongly differs and can impact the properties of these fluids of light. To verify that these variations do not change significantly the long-wavelength behavior of $\Delta S$, we numerically studied the dependence on four key parameters: (i) the losses $\alpha$, (ii) the width of the pump beam $w_{0, y}$, (iii) the nonlocality, and the (iv) saturation of the nonlinear response.
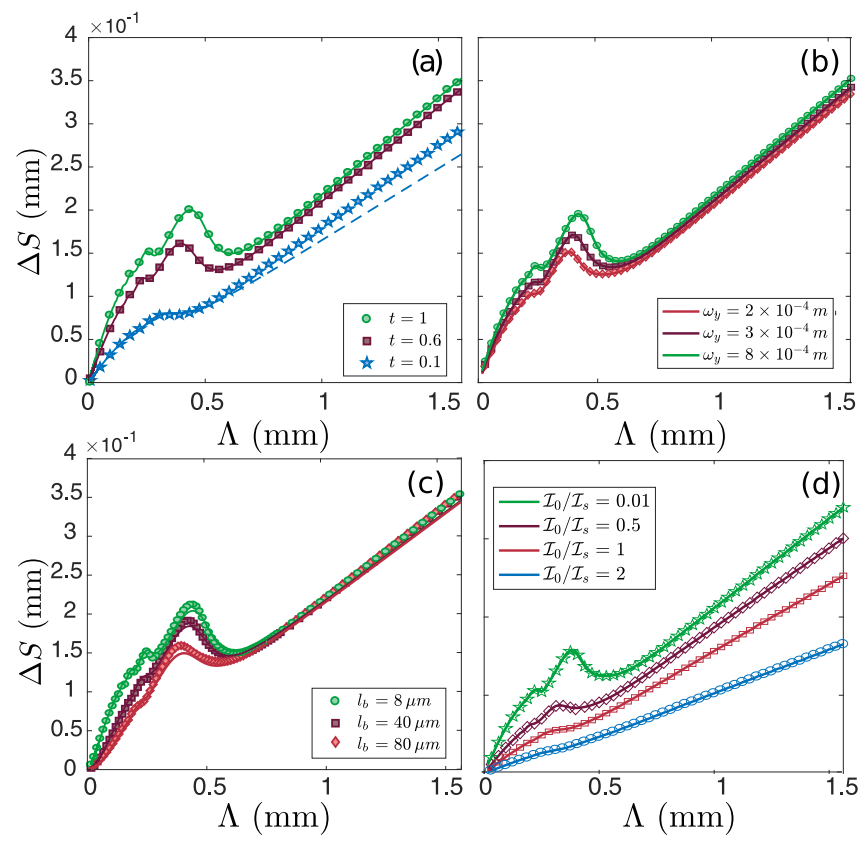

FIG. 5. Numerical simulations (symbols) and analytical solutions (solid and dashed line) of $\Delta S$ as a function of $\Lambda$. (a) Different cell transmissions $t$. The simulations and the theory model are similar as long as the transmission remains large $(t>0.5)$. (b) Different background widths $w_{0, y}$. (c) Different nonlocal transport length scales $l_{b}$. The oscillations are smoothed by nonlocality. In our system $l_{b}<10 \mu \mathrm{m}$. (d) Different saturation intensities $\mathcal{I}_{s}$ of the nonlinear Kerr interaction. Once again oscillations are smoothed by a saturation of the medium. For all the simulations $\Delta n=1.0 \times 10^{-5}$.

All the simulations have been performed using a secondorder split-step method on the 2D nonlinear Schrödinger equation and a common set of parameters. The background intensity is set to $\rho_{0}=2.5 \times 10^{5} \mathrm{~W} / \mathrm{m}^{2}$, the linear index is set to $n=1$, and the nonlinear index is set to $n_{2}=4 \times 10^{-11}$ $\mathrm{m}^{2} / \mathrm{W}$. In the lossless situation, the nonlinear change of refractive index is equal to $\Delta n=1.0 \times 10^{-5}$. Typical interactions accessible in our experimental configurations are between $\Delta n=1.0 \times 10^{-4}$ and $1.0 \times 10^{-7}$. The simulation results are presented in Fig. 5.

In Fig. 5(a), the displacement $\Delta S$ is plotted, for different cell transmissions $t=\exp (-\alpha L)$. The colored points stem from numerical simulations whereas the theoretical curves are plotted in black solid. A full derivation of the analytical model is given in the Appendices A and B. Absorption smooths out the oscillations at small $\Lambda$, similarly to a reduction of the nonlinear interactions $\Delta n$ as seen in Fig. 2. However the long- $\Lambda$ limit is qualitatively unchanged from the lossless case. The analytical predictions (dashed lines) give an accurate estimation of the long- $\Lambda$ slope for transmission larger than 0.5 .

In Fig. 5(b), the effect of the finite beam width $w_{0, y}$ on the displacement $\Delta S$ is studied. We notice a reduction in the displacement oscillations amplitude when $w_{0, y}$ decreases. Similarly to the absorption, this effect does not affect the general shape of the displacement curve and its large $\Lambda$ linear trend. It can be understood intuitively, because for smaller beam width $w_{0, y}$ the Kerr self-defocusing effect increases and 
therefore the background density spreads faster in the transverse plane along the propagation. This results in a decrease of the beam intensity on the major axis during the propagation and a consequent reduction of the effective interaction $\Delta n$.

In Fig. 5(c), the impact of nonlocality is reported. The nonlinear phase shift formula (17) has been generalized using the nonlocal dispersion relation to take ballistic transport of excited atoms into account in the theory (see the Appendix C for details). The theoretical predictions are plotted in black solid line and match perfectly with simulations. The main effect here is more subtle than the ones of the losses or the finite width of the beam. The slope of the linear trend at high $\Lambda$ remains unchanged but a significant modification of the displacement in the oscillating part is observed. This effect becomes significant for nonlocal ballistic length scales $l_{b}$ much longer than the typical ones of atomic vapors (typically, $l_{d} \approx 8 \mu \mathrm{m}$ at $400 \mathrm{~K}$ ). The situation is very different in the thermo-optic media considered in Ref. [15], where the nonlocal length is on the order of $100 \mu \mathrm{m}$ [12] and thus is able to significantly modify the behavior of the displacement for small $\Lambda$.

Finally, in Fig. 5(d), we have studied the impact of a saturation of the nonlinearity. The interaction strength $\Delta_{n}$ is replaced by $\Delta n \times \frac{1}{1+I / \mathcal{I}_{s}}$, where $\mathcal{I}_{s}$ is the saturation intensity. This model reproduces saturation observed in atomic media and photorefractive crystals. Compared to losses, finite beam width, and nonlocality, the effect of saturation on the displacement is the most important, as it not only attenuates the oscillations at small $\Lambda$ but also modifies the slope at large $\Lambda$. This correction is a consequence of the reduction of the sound velocity by a larger factor to $c_{s} \times \frac{1}{\left(1+I / \mathcal{I}_{s}\right)^{2}}$. Nevertheless, saturation does not lead to a constant value for the large- $\Lambda$ limit predicted by the geometrical approach.

All these simulations confirm that the corrections to the ideal lossless model are able to modify the behavior of $\Delta S$ at small $\Lambda$, but do not affect the linear trend at large $\Lambda$. The impact of the interferences between Bogoliubov modes is therefore robust and can thus be envisioned as a tool to probe the dispersion and the static structure factor of the photon fluid, in a similar way to what was done with atomic BEC [22]. In the last part of this paper, we propose an explanation for the robustness of these interferences and for their importance to understand the superfluid behavior based on a universal mechanism known as the Sakharov oscillations [19,21].

\section{B. Stimulated Sakharov-like oscillations}

The Bogoliubov excitation [Eq. (4)] generated at the entrance of the nonlinear medium consists in a superposition of counterpropagating plane waves in the $\mathbf{r}_{\perp}$ plane with opposite wave vectors $\mathbf{k}_{\perp}$ and $-\mathbf{k}_{\perp}$. These Bogoliubov components are simultaneously generated at the medium entrance and oscillate at the respective angular frequencies $\Omega_{\mathrm{B}}\left(\mathbf{k}_{\perp}\right)$ and $-\Omega_{\mathrm{B}}\left(\mathbf{k}_{\perp}\right)$ along the propagation axis, which is analogous to time. As a consequence, at a given effective time $z$, these components will have acquired a relative phase difference of $2 \Omega_{\mathrm{B}}\left(\mathbf{k}_{\perp}\right) z$. Interestingly, this behavior is very similar to the one predicted for the Sakharov oscillations in cosmology $[19,20]$ and can be understood in terms of the interference between the counterpropagating phonons that are spontaneously
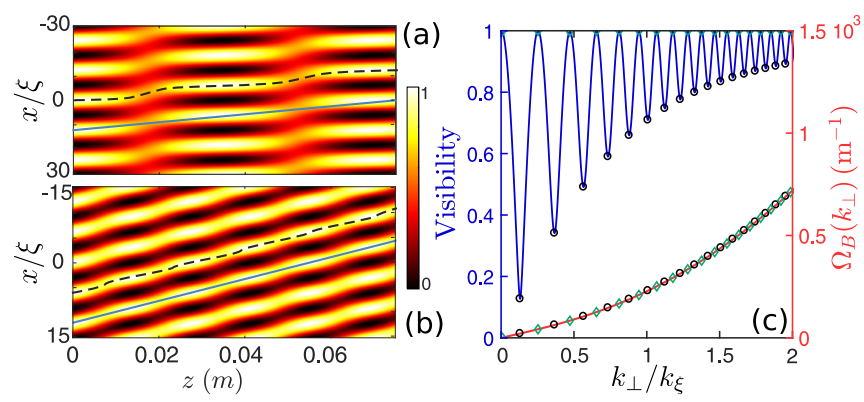

FIG. 6. Evolution along the $z$ axis of the transverse field intensity in a given $y$ plane for (a) $k_{\perp} \xi=0.5$ and (b) $k_{\perp} \xi=1$. The background intensity is subtracted on both images. The black dashed curves follow the center of a bright fringe. The blue solid line is a trajectory of a Bogoliubov mode at the speed of sound. (c) Visibility of the interference fringes at the output plane $z=L$ as a function of $k_{\perp}$ (solid blue line). Visibility maxima are shown by green diamonds and minima are shown by black circles. The dispersion relation (solid red line, right axis) is reconstructed using a sampling based on the position of the maxima $\left[\Omega_{\mathrm{B}}\left(\mathbf{k}_{\perp}\right)=p \pi / L\right]$ and minima $\left[\Omega_{\mathrm{B}}\left(\mathbf{k}_{\perp}\right)=\right.$ $(p+1 / 2) \pi / L]$. Here $\Delta n=1.0 \times 10^{-5}$.

generated after a quantum quench $[21,34,35]$. Here, we draw the analogy and we consider our experimental observations as a stimulated analog of the Sakharov-like oscillations by seeding phonons on the $+\mathbf{k}_{\perp}$ mode.

For paraxial fluids of light experiments, we only have access to the intensity at $z=L$ and not inside the medium. Therefore, we have solved numerically the nonlinear Schrödinger equation [Eq. (1)] and computed the intensity of the total electric field inside the nonlinear medium at every transverse plane along the $z$ axis to evidence the stimulated Sakharov-like oscillations in this optical system. In Fig. 6, we present the intensity profiles along $x$ in both the phonon regime $\left(k_{\perp} \xi \leqslant 1\right)$ in Fig. $6($ a) and the free-particle regime $\left(k_{\perp} \xi>1\right)$ in Fig. 6(b). In this figure, the background fluid density has been subtracted. Two remarkable observations can be highlighted in Fig. 6.

First, constructive (maximum contrast) or destructive (minimum contrast) interferences between the counterpropagating Bogoliubov waves are clearly visible in the transverse direction. Along $z$, constructive interferences are located at $\Omega_{\mathrm{B}}\left(\mathbf{k}_{\perp}\right) z=p \pi$, with $p \geqslant 1$ integers valued. In contrast, when $\Omega_{\mathrm{B}}\left(\mathbf{k}_{\perp}\right) z=(p+1 / 2) \pi$, Bogoliubov modes destructively interfere and the contrast is minimum. Interestingly, these interference patterns can also be observed at a fixed effective time (e.g., $z=L$ ) by changing the value of $\mathbf{k}_{\perp}$. In Fig. 6(c), we have extracted the dispersion relation using this approach. At fixed $z=L$, we reported each value of $\mathbf{k}_{\perp}$ leading to a visibility maximum, as we know that $\Omega_{\mathrm{B}}\left(\mathbf{k}_{\perp}\right)=p \pi / L$ [green diamonds in Fig. 6(c)]. To increase the resolution of the reconstruction we can apply the same procedure with the visibility minima [black circles in Fig. 6(c)] and obtain a sampling of the dispersion for $\Omega_{\mathrm{B}}\left(\mathbf{k}_{\perp}\right)=(p+1 / 2) \pi / L$.

Second, we can notice that the reduction in the contrast of the interference fringes that is observed when the two Bogoliubov components destructively interfere is more pronounced at low [Fig. 6(a)] than at large [Fig. 6(b)] wave vectors. This effect is not present in the spontaneous Sakharov oscillations triggered by zero-point fluctuations [21] and is a 
direct consequence of the stimulation of the process by the classical incident field in the $+\mathbf{k}_{\perp}$ mode.

Indeed, by seeding the process we break the symmetry between $+\mathbf{k}_{\perp}$ and $-\mathbf{k}_{\perp}$ modes, so the visibility reduction can be understood by comparing $\left|u^{2}\left(\mathbf{k}_{\perp}\right)\right|$ and $\left|v^{2}\left(\mathbf{k}_{\perp}\right)\right|$ using (6). When $k_{\perp} \xi \gg 1$, then $\left|v^{2}\left(\mathbf{k}_{\perp}\right)\right|$ becomes small comparing to $\left|u^{2}\left(\mathbf{k}_{\perp}\right)\right|$ and therefore the interference contrast is reduced. As a consequence, we can see in Fig. 6 that the trajectories of a bright fringe (black dashed line) are much less deformed with respect to the speed of sound propagation (blue solid line) for $k_{\perp} \xi=1$ [Fig. 6(b)] than for $k_{\perp} \xi=0.5$ [Fig. 6(a)], where a staircaselike structure is apparent. This exemplifies once again why the geometrical approach is a good approximation only in the free-particle regime $\left(k_{\perp} \xi>1\right)$.

\section{CONCLUSION}

In this paper, we have studied the Bogoliubov excitations of a photon superfluid. We have experimentally demonstrated a previously hidden phenomenon whereby the propagation of plane-wave excitations in the fluid does not tend to the geometric prediction for the displacement, namely, the product of the sound velocity $c_{s}$ by the effective time $L$, but keeps growing linearly with the excitation wavelength. This is shown to be a direct consequence of the interference between counterpropagating Bogoliubov modes that are generated at an interaction quench and have only been observed in atomic superfluids [36]. These interferences can also be interpreted as stimulated Sakharov oscillations [21], i.e., an analog of fluctuations imprinted in the primordial universe and visible as oscillations in the cosmic microwave background power spectrum $[19,20]$. These results shows that these interferences are an essential element to describe accurately the dynamics of excitations in superfluids of light in the classical regime. It brings a different understanding of superfluidity for paraxial fluids of light and the next generation of experiments can now be conducted without the external classical excitations (no probe beam). In this configuration, the laser intrinsic noise (the spatial shot noise) will trigger the generation of phonons in the fluid. By measuring the spatial noise spectrum (the static structure factor) it will then become possible to probe the spontaneous creation of phonons due to the quantum fluctuation and possibly observe quantum depletion.

\section{ACKNOWLEDGMENTS}

This work has received funding from the French Agence Nationale de la Recherche (ANR) grant (C-FLigHT No. 138678-ANR-16-ACHN-0027 and Quantum Fluids of Light QFL grant No. ANR-16-CE30-0021) and from the European Union's Horizon 2020 Research and Innovation Program under Grant No. 820392 (PhoQuS). Q.G. and A.B. thank the Institut Universitaire de France (IUF) for support. I.C. acknowledges support from the Provincia Autonoma di Trento.

\section{APPENDIX A: EXPERIMENTAL ALIGNMENT PROCEDURE}

In order to accurately measure the displacement $\Delta S$, one needs to precisely align the reference beam with respect to the high-power one. The alignment procedure is as follows.
(1) We first make sure that both background beams (probe off) roughly propagate with the same transverse wave vector and are correctly positioned one above the other (their respective center should lie on the same vertical axis).

(2) We then switch the probe beam on. The next step is to align the interference fringes of the lower and upper interference patterns. We start by removing the cell and make sure that bright fringes on the bottom face bright fringes on the top. Of course, by doing so, the optical axes of the lower and upper beams are not parallel anymore. We should then switch to $k$ space, bring back the backgrounds to the initial position $\left(k_{\perp}=0\right)$, and repeat this procedure iteratively (beam walking). We finally check that for every transverse wave vector $k_{\perp}$ the interference fringes remain aligned before putting the cell back on the beam path.

\section{APPENDIX B: PHOTON ABSORPTION}

Photon absorption is described in (1) by the term proportional to $\alpha \geqslant 0$. When $\alpha \neq 0$, the $\mathbf{r}_{\perp}$-independent electricfield envelope $\mathcal{E}_{0}$ and its linearized fluctuations $\delta \mathcal{E}$ acquire the following $z$ dependences:

$$
\begin{aligned}
& \mathcal{E}_{0}(z)=\sqrt{\rho_{0}} e^{-\alpha z / 2-i k_{0} \Delta n\left(1-e^{-\alpha z}\right) / \alpha} \\
\delta \mathcal{E}\left(\mathbf{r}_{\perp}, z\right)= & e^{-i k_{0} \Delta n\left(1-e^{-\alpha z}\right) / \alpha} \\
& \times \int \frac{d^{2} \mathbf{k}_{\perp}}{(2 \pi)^{2}}\left\{u\left(\mathbf{k}_{\perp}, z\right) b_{\mathbf{k}_{\perp}} e^{i\left[\mathbf{k}_{\perp} \cdot \mathbf{r}_{\perp}-\int_{0}^{z} d z^{\prime} \Omega_{\mathrm{B}}\left(\mathbf{k}_{\perp}, z^{\prime}\right)\right]}\right. \\
& \left.+v^{*}\left(\mathbf{k}_{\perp}, z\right) b_{\mathbf{k}_{\perp}}^{*} e^{-i\left[\mathbf{k}_{\perp} \cdot \mathbf{r}_{\perp}-\int_{0}^{z} d z^{\prime} \Omega_{\mathrm{B}}^{*}\left(\mathbf{k}_{\perp}, z^{\prime}\right)\right]}\right\}
\end{aligned}
$$

In Eqs. (B1) and (B2), $\rho_{0}$ is the density of the paraxial fluid of light at $z=0$ and $\Delta n=g \rho_{0} / k_{0}$ is the corresponding nonlinearity. We treat the $z$ dependence of the Bogoliubov spectrum $\Omega_{\mathrm{B}}$ and of the Bogoliubov amplitudes $u$ and $v$ in the adiabaticevolution approximation $[17,37]$. Searching for real-valued $u$ and $v$ such that $u^{2}-v^{2}=1$ for all $z$, this gives

$$
\begin{gathered}
\Omega_{\mathrm{B}}\left(\mathbf{k}_{\perp}, z\right)=\sqrt{\frac{k_{\perp}^{2}}{2 k_{0}}\left(\frac{k_{\perp}^{2}}{2 k_{0}}+2 k_{0} \Delta n e^{-\alpha z}\right)}-\frac{i \alpha}{2}, \\
u\left(\mathbf{k}_{\perp}, z\right) \pm v\left(\mathbf{k}_{\perp}, z\right)=\left\{\frac{k_{\perp}^{2}}{2 k_{0}} / \operatorname{Re}\left[\Omega_{\mathrm{B}}\left(\mathbf{k}_{\perp}, z\right)\right]\right\}^{ \pm \frac{1}{2}} .
\end{gathered}
$$

All the observables computed in this paper rely on the input-output relation (24), which also holds when $\alpha \neq 0$ provided (25) and (26) are, respectively, replaced with

$$
\begin{aligned}
U\left(\mathbf{k}_{\perp}\right)= & u\left(\mathbf{k}_{\perp}, 0\right) u\left(\mathbf{k}_{\perp}, L\right) e^{-i \int_{0}^{L} d z \Omega_{\mathrm{B}}\left(\mathbf{k}_{\perp}, z\right)} \\
- & v\left(\mathbf{k}_{\perp}, 0\right) v\left(\mathbf{k}_{\perp}, L\right) e^{i \int_{0}^{L} d z \Omega_{\mathrm{B}}^{*}\left(\mathbf{k}_{\perp}, z\right)}, \\
V\left(\mathbf{k}_{\perp}\right)= & u\left(\mathbf{k}_{\perp}, 0\right) v\left(\mathbf{k}_{\perp}, L\right) e^{-i \int_{0}^{L} d z \Omega_{\mathrm{B}}\left(\mathbf{k}_{\perp}, z\right)} \\
& -v\left(\mathbf{k}_{\perp}, 0\right) u\left(\mathbf{k}_{\perp}, L\right) e^{i \int_{0}^{L} d z \Omega_{\mathrm{B}}^{*}\left(\mathbf{k}_{\perp}, z\right)} .
\end{aligned}
$$

For example, the nonlinear phase $\Phi_{\mathrm{NL}}\left(\mathbf{k}_{\perp}\right)$ expected for $\alpha \neq$ 0 reads

$$
\begin{aligned}
\Phi_{\mathrm{NL}}\left(\mathbf{k}_{\perp}\right) & \\
= & \arctan \left(\frac{\left[k_{\perp}^{2} /\left(2 k_{0}\right)\right]^{2}+\operatorname{Re}\left[\Omega_{\mathrm{B}}\left(\mathbf{k}_{\perp}, 0\right)\right] \operatorname{Re}\left[\Omega_{\mathrm{B}}\left(\mathbf{k}_{\perp}, L\right)\right]}{k_{\perp}^{2} /\left(2 k_{0}\right) \times\left\{\operatorname{Re}\left[\Omega_{\mathrm{B}}\left(\mathbf{k}_{\perp}, 0\right)\right]+\operatorname{Re}\left[\Omega_{\mathrm{B}}\left(\mathbf{k}_{\perp}, L\right)\right]\right\}}\right. \\
& \left.\times \tan \left\{\int_{0}^{L} d z \operatorname{Re}\left[\Omega_{\mathrm{B}}\left(\mathbf{k}_{\perp}, z\right)\right]\right\}\right),
\end{aligned}
$$


from which we infer the following linear trend of the transverse displacement $\Delta S(\Lambda)$ in the long-wavelength, superfluid regime:

$$
\Delta S(\Lambda) \simeq \frac{1}{2 \pi} \arctan \left(2 k_{0} \Delta n L \times \frac{2}{\alpha L} \frac{1-e^{-\alpha L / 2}}{1+e^{-\alpha L / 2}}\right) \Lambda .
$$

\section{APPENDIX C: NONLOCALITY MODEL}

So far, we have assumed that the nonlinear change of refractive index $\Delta n\left(\mathbf{r}_{\perp}\right)$ at a given position $\mathbf{r}_{\perp}$ in the transverse plane only depends on the laser intensity at this point, $\propto\left|\mathcal{E}\left(\mathbf{r}_{\perp}\right)\right|^{2}$, and not on the intensity nearby. However, such a local dielectric response may not correctly describe hot atomic vapors, in which the ballistic transport of excited atoms on large length scales induces nonlocality [38]. Indeed, the coherence between the ground and excited states of an atom, from which the medium nonlinear response arises, is more likely to be transported away in hot vapors, as the atomic motion is more significant at large temperatures.

Following Ref. [38], we can express the nonlocal nonlinear change of refractive index $\Delta n^{\mathrm{nl}}\left(\mathbf{r}_{\perp}\right)$ as follows:

$$
\Delta n^{\mathrm{nl}}\left(\mathbf{r}_{\perp}\right)=n_{2} \int d^{2} \mathbf{r}_{\perp}^{\prime} G_{\mathrm{b}}\left(\mathbf{r}_{\perp}-\mathbf{r}_{\perp}^{\prime}\right)\left|\mathcal{E}\left(\mathbf{r}_{\perp}^{\prime}\right)\right|^{2},
$$

where $G_{\mathrm{b}}$ stands for the steady-state ballistic response function. By using the convolution theorem, we can then easily rewrite the Bogoliubov dispersion relation (6) in the nonlocal case:

$$
\Omega_{\mathrm{B}}^{\mathrm{nl}}\left(\mathbf{k}_{\perp}\right)=\sqrt{\frac{k_{\perp}^{2}}{2 k_{0}}\left[\frac{k_{\perp}^{2}}{2 k_{0}}+2 k_{0}\left|n_{2}\right| \rho_{0} \widetilde{G}_{\mathrm{b}}\left(\mathbf{k}_{\perp}\right)\right]},
$$

where $\widetilde{G}_{\mathrm{b}}$ is the Fourier transform of $G_{\mathrm{b}}$. By introducing the ballistic transport length scale $\ell_{\mathrm{b}}=u \tau$-where $u=$ $\sqrt{2 k_{\mathrm{B}} T / m}$ is the most probable speed of the atoms in the transverse plane (at the vapor temperature $T$ ) and $\tau=2 / \gamma$ is the characteristic decoherence time-and by calling erfc the complementary error function, $\widetilde{G}_{\mathrm{b}}$ can be written in the following way:

$$
\widetilde{G}_{\mathrm{b}}\left(\mathbf{k}_{\perp}\right)=\sqrt{\pi} \frac{e^{1 /\left(k_{\perp} \ell_{\mathrm{b}}\right)^{2}}}{k_{\perp} \ell_{\mathrm{b}}} \operatorname{erfc}\left(\frac{1}{k_{\perp} \ell_{\mathrm{b}}}\right) .
$$

The solid lines in Fig. 5(c) have been obtained by plugging (C2) into (28). In the experiment, the vapor temperature was $400 \mathrm{~K}$, leading to a nonlocal ballistic length $\ell_{\mathrm{b}}$ of about $8 \mu \mathrm{m}$. As can be seen in Fig. 5(c), nonlocal effects do not significantly affect the shift $\Delta S$ for such a small value of $\ell_{\mathrm{b}}$.
[1] N. Bogoliubov, On the theory of superfluidity, J. Phys. 11, 23 (1947).

[2] L. Pitaevskii and S. Stringari, Bose-Einstein Condensation and Superfluidity, International Series of Monographs on Physics (Oxford University, New York, 2016), Vol. 164.

[3] A. J. Leggett, Bose-Einstein condensation in the alkali gases: Some fundamental concepts, Rev. Mod. Phys. 73, 307 (2001).

[4] I. Carusotto and C. Ciuti, Quantum fluids of light, Rev. Mod. Phys. 85, 299 (2013).

[5] J. Kasprzak, M. Richard, S. Kundermann, A. Baas, P. Jeambrun, J. M. J. Keeling, F. M. Marchetti, M. H. Szymańska, R. André, J. L. Staehli, V. Savona, P. B. Littlewood, B. Deveaud, and L. S. Dang, Bose-Einstein condensation of exciton polaritons, Nature (London) 443, 409 (2006).

[6] J. Klaers, J. Schmitt, F. Vewinger, and M. Weitz, Bose-einstein condensation of photons in an optical microcavity, Nature (London) 468, 545 (2010).

[7] Y. Pomeau and S. Rica, Diffraction non linéaire, C. R. Acad. Sci. Ser. B 317, 1287 (1993).

[8] I. Carusotto, Superfluid light in bulk nonlinear media, Proc. R. Soc. A 470, 20140320 (2014).

[9] C. Noh and D. G. Angelakis, Quantum simulations and manybody physics with light, Rep. Prog. Phys. 80, 016401 (2016).

[10] W. Wan, S. Jia, and J. W. Fleischer, Dispersive superfluid-like shock waves in nonlinear optics, Nat. Phys. 3, 46 (2007).

[11] C. Michel, O. Boughdad, M. Albert, P.-É. Larré, and M. Bellec, Superfluid motion and drag-force cancellation in a fluid of light, Nat. Commun. 9, 2108 (2018).

[12] D. Vocke, K. Wilson, F. Marino, I. Carusotto, E. M. Wright, T. Roger, B. P. Anderson, P. Öhberg, and D. Faccio, Role of geometry in the superfluid flow of nonlocal photon fluids, Phys. Rev. A 94, 013849 (2016).
[13] M. Elazar, S. Bar-Ad, V. Fleurov, and R. Schilling, An alloptical event horizon in an optical analogue of a laval nozzle, Lect. Notes Phys. 870, 275 (2013).

[14] N. Šantić, A. Fusaro, S. Salem, J. Garnier, A. Picozzi, and R. Kaiser, Nonequilibrium Precondensation of Classical Waves in Two Dimensions Propagating Through Atomic Vapors, Phys. Rev. Lett. 120, 055301 (2018).

[15] D. Vocke, T. Roger, F. Marino, E. M. Wright, I. Carusotto, M. Clerici, and D. Faccio, Experimental characterization of nonlocal photon fluids, Optica 2, 484 (2015).

[16] Q. Fontaine, T. Bienaimé, S. Pigeon, E. Giacobino, A. Bramati, and Q. Glorieux, Observation of the Bogoliubov Dispersion in a Fluid of Light, Phys. Rev. Lett. 121, 183604 (2018).

[17] P.-E. Larré, S. Biasi, F. Ramiro-Manzano, L. Pavesi, and I. Carusotto, Pump-and-probe optical transmission phase shift as a quantitative probe of the Bogoliubov dispersion relation in a nonlinear channel waveguide, Eur. Phys. J. D 71, 146 (2017).

[18] T. D. Ferreira, N. A. Silva, and A. Guerreiro, Superfluidity of light in nematic liquid crystals, Phys. Rev. A 98, 023825 (2018).

[19] A. D. Sakharov, The initial stage of an expanding Universe and the appearance of a nonuniform distribution of matter, Sov. Phys. JETP 22, 241 (1966).

[20] A. R. Liddle and D. H. Lyth, Cosmological Inflation and LargeScale Structure (Cambridge University, Cambridge, England, 2000).

[21] C.-L. Hung, V. Gurarie, and C. Chin, From cosmology to cold atoms: Observation of Sakharov oscillations in a quenched atomic superfluid, Science 341, 1213 (2013).

[22] I. Shammass, S. Rinott, A. Berkovitz, R. Schley, and J. Steinhauer, Phonon Dispersion Relation of an Atomic BoseEinstein Condensate, Phys. Rev. Lett. 109, 195301 (2012).

[23] R. W. Boyd, Nonlinear Optics (Elsevier, Amsterdam, 2003). 
[24] P.-É. Larré and I. Carusotto, Propagation of a quantum fluid of light in a cavityless nonlinear optical medium: General theory and response to quantum quenches, Phys. Rev. A 92, 043802 (2015).

[25] P.-É. Larré and I. Carusotto, Prethermalization in a quenched one-dimensional quantum fluid of light: Intrinsic limits to the coherent propagation of a light beam in a nonlinear optical fiber, Eur. Phys. J. D 70, 45 (2016).

[26] P.-É. Larré, D. Delande, and N. Cherroret, Postquench prethermalization in a disordered quantum fluid of light, Phys. Rev. A 97, 043805 (2018).

[27] Q. Glorieux, R. Dubessy, S. Guibal, L. Guidoni, J.-P. Likforman, T. Coudreau, and E. Arimondo, Double- $\lambda$ microscopic model for entangled light generation by four-wave mixing, Phys. Rev. A 82, 033819 (2010).

[28] Q. Glorieux, J. B. Clark, N. V. Corzo, and P. D. Lett, Generation of pulsed bipartite entanglement using four-wave mixing, New J. Phys. 14, 123024 (2012).

[29] I. H. Agha, C. Giarmatzi, Q. Glorieux, T. Coudreau, P. Grangier, and G. Messin, Time-resolved detection of relative-intensity squeezed nanosecond pulses in an ${ }^{87} \mathrm{Rb}$ vapor, New J. Phys. 13, 043030 (2011).

[30] S. Reynaud, A. Heidmann, E. Giacobino, and C. Fabre, I quantum fluctuations in optical systems, Prog. Opt. 30, 1 (1992).
[31] J.-M. Courty and S. Reynaud, Generalized linear input-output theory for quantum fluctuations, Phys. Rev. A 46, 2766 (1992).

[32] Q. Fontaine, H. Hu, S. Pigeon, T. Bienaimé, E. Wu, E. Giacobino, A. Bramati, and Q. Glorieux, Attenuation-free nondiffracting bessel beams, Opt. Express 27, 30067 (2019).

[33] O. Boughdad, A. Eloy, F. Mortessagne, M. Bellec, and C. Michel, Anisotropic nonlinear refractive index measurement of a photorefractive crystal via spatial self-phase modulation, Opt. Express 27, 30360 (2019).

[34] G. I. Martone, P.-É. Larré, A. Fabbri, and N. Pavloff, Momentum distribution and coherence of a weakly interacting Bose gas after a quench, Phys. Rev. A 98, 063617 (2018).

[35] S. Robertson, F. Michel, and R. Parentani, Controlling and observing nonseparability of phonons created in time-dependent 1D atomic Bose condensates, Phys. Rev. D 95, 065020 (2017).

[36] M. Cheneau, P. Barmettler, D. Poletti, M. Endres, P. Schauß, T. Fukuhara, C. Gross, I. Bloch, C. Kollath, and S. Kuhr, Lightcone-like spreading of correlations in a quantum many-body system, Nature (London) 481, 484 (2012).

[37] M. Born and V. Fock, Beweis des adiabatensatzes, Z. Phys. 51, 165 (1928).

[38] S. Skupin, M. Saffman, and W. Królikowski, Nonlocal Stabilization of Nonlinear Beams in a Self-Focusing Atomic Vapor, Phys. Rev. Lett. 98, 263902 (2007). 